\title{
Research \& Application of Ash Condensation Analysis Technology
}

\author{
Jianbo Zhan, Hao Wang, Han Zheng*, Jiao Xie, Zhenhua Yu, Ying Zhang, Tingting Yu, Baoshan Yue, Xu Wang, and \\ Geng Li \\ R\&D Centre, China Tobacco Yunnan Industrial Co., Ltd. 650231 Kunming, Yunnan, P.R.China
}

\begin{abstract}
On the basis of self-developed cigarette ash analysis software, through the calculation of the cigarette ash index, the ash condensation ability of the different types of cigarettes was analyzed in this paper. The results show that the cigarette ash index has the advantages of simple calculation, quick response of ash analysis software, small coefficient of variation between different cigarette ash condensation index, among different samples there is no obvious little difference in ash condensation index. Therefore, the cigarette ash condensation index can be used as an important index and effective means to measure the performance of the cigarette ash, meanwhile, the index can be applied to the comprehensive assessment of the ash condensation performance of the cigarette paper.
\end{abstract}

\section{Introduction}

Cigarette paper is one of the main materials during the making process of cigarettes, which affects significantly not only on the packaging of tobacco leaf, cigarettes' appearance, combustion velocity and smoldering, but also on sensory quality and chemical composition of smoke[1-3]. Cigarette paper not only carries the key image and connotation of the cigarette products, but also has a special and direct influence on the cigarette smoke due to its involvement in combustion. On the one hand, the gas composition after combustion of cigarette paper is an important component of cigarette smoke, which will be together smoked with the mainstream smoke; on the other hand, the intrinsic quality of cigarette paper will also affect the combustion status of cigarettes, which plays a decisive role in ash appearance and grey degree of cigarette paper after combustion. How to improve the sensory quality and integrity of ash are double major problems of cigarette paper[4-9].

After combustion, the ideal cigarette ash appearance should show no obvious cracks or tilt parts, and during the smoking process, the ash should not break[10,11]. The excellent cigarette ash performance mainly presents as perfect smoke appearance, and after cigarettes' combustion the ash is still bonded together with high integrity degree. The ideal ash is the process of full combustion, and the ash is white but not scattered. So far, there is no systematic solution and evaluation system on how to evaluate the capability of cigarette ash appearance and ash integration, and on how to optimize the performance of cigarette ash.

In order to further study the performance of cigarette ash and cigarette paper, ash analysis system with independent research and development was applied, and the concept of cigarette ash index was put forward in this paper. Based on the appearance of the samples' combustion observation and comparison of different cigarettes' ash index, calculation of cigarette ash index different samples from the same batch was completed, and the feasibility of applying ash index as reflection of cigarette paper ash properties was verified. The application prospect of cigarette ash analysis system and influencing factors of cigarette ash integrity were analyzed.

\section{Experimental materials and methods}

KBF240 constant temperature and humidity incubator (German Binder company) was mainly used for the balance of cigarette samples before combustion, and the cigarette samples were placed in constant temperature and humidity incubator with temperature $(22+1) \mathrm{C}$ for $48 \mathrm{~h}$, and the balance moisture is set as $20 \%+2 \%$.

The cigarette ash analysis system YNZY-JYNHV2.1, an independent design and development of China Tobacco Yunnan Industrial Co., Ltd., was applied. The system is mainly composed of a camera module and a computer analysis module. The camera module is mainly designed to capture the instantaneous image capture of cigarette ash, and the computer analysis module mainly completes the analysis of the captured image and calculates the coagulation index.

Experimental cigarettes from a certain brand of cigarettes were purchased from markets and the cigarettes were numbered $1 \#$ to $6 \#$ separately.

\section{Results and discussion}

\footnotetext{
* Corresponding author: 93320213@qq.com
} 
Table 1 Ash condensation indexes of different samples

\begin{tabular}{lllllll}
\hline Samples & $1 \#$ & $2 \#$ & $3 \#$ & $4 \#$ & $5 \#$ & $6 \#$ \\
\hline AI $(\%)$ & 93.5 & 99.1 & 98.9 & 97.6 & 90.7 & 88.2 \\
\hline
\end{tabular}

\subsection{Ash Condensation Analysis}

Fig. 1 shows the different ash condensation grades of cigarette paper coagulation effect (from left to right were samples 1 \# to 6 \#). As can be seen from Fig. 1, there is a certain difference in ash condensation among different brands of cigarette paper, and qualitative analysis of the ash condensation quality of cigarettes could be made. Cheng Zhangang et al insisted that the slump tightness directly affect on the cigarette ash capacity, and the amount and size of ash fragments have a direct impact on the ash fall during the smoking process, which is the decisive factor of ash condensation capacity [12]. Cracking of cigarettes and ash smoothness are potential factors in determining the quality of cigarettes. In the definition of ash capacity in some references, the appearance factor accounts for $75 \%$. However, the calculation and determination of the ash capacity still need to be subjective for score calculation of different properties, and the scale and scoring are influenced largely by the subjective factors of judges. Therefore, how to quantify objectively and accurately the cohesion performance of different cigarette paper has always been the focus to determine the ash condensation capacity of cigarettes.

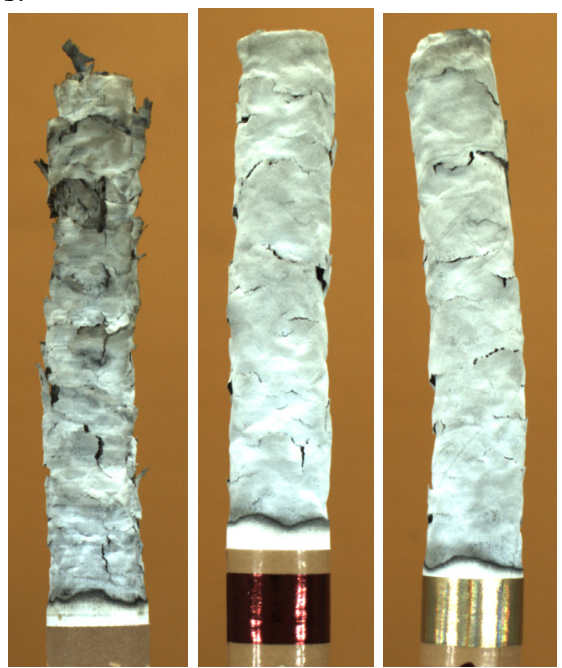

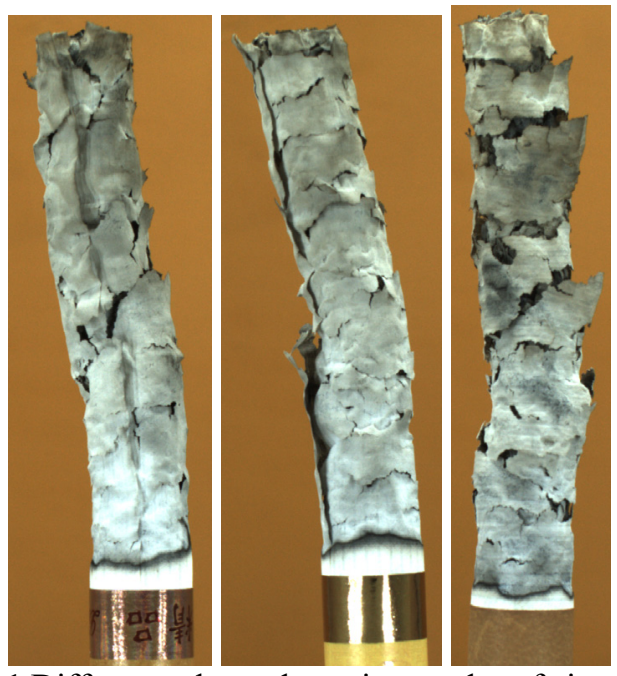

Fig.1 Different ash condensation grades of cigarette paper(Samples1\#-6\#)

\subsection{Calculation of Ash Condensation Index and Analysis on Variation Coefficient}

Ash Condensation Index AI was defined by authors in the process of ash condensation. In Fig. 2, for example, the ash condensation index $\mathrm{AI}$ is the ratio of the area of the uncracked area to the total area after the image recognition[13]

$$
A I=\left(1-\frac{S_{r}}{S_{t}}\right) \times 100 \%
$$

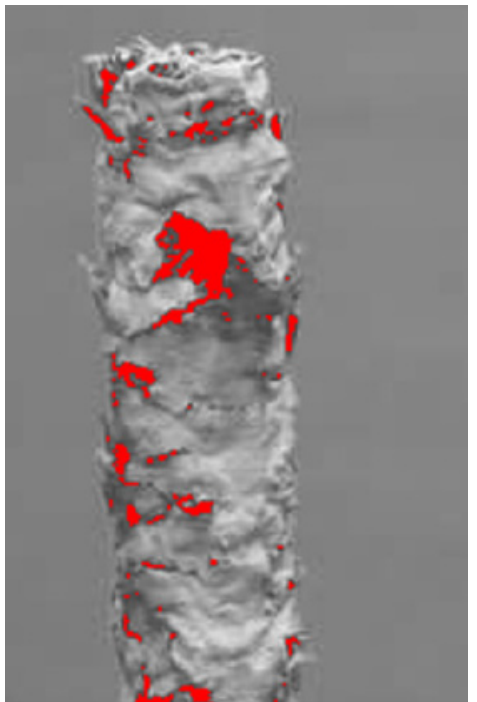

Fig.2 Ash condensation calculation

It is not difficult to find that the greater the ash condensation index, the better the ash condensation effect is after comparing the data in Table 1 and the ash appearance results in Fig. 1. The index AI can intuitively and accurately reflect the ash condensation ability. 
In order to further investigate the difference among the ash condensation index of different samples in the same batch, authors calculated the variation coefficient of the ash condensation index $\mathrm{CV}$ as follows[13]:

$\mathrm{CV}=\mathrm{SD} / \mathrm{MN}^{*} 100 \%$

Where SD is the standard deviation of the same set of samples and $\mathrm{MN}$ is the standard value for the same batch of different samples. Each group of samples was measured five times and the corresponding standard deviation, mean value and variation coefficient of were calculated as shown in Table 2.

It can be seen from Table 2 that the variation coefficient of different samples from the same brand is less than $3 \%$, which indicates that the ash condensation index of the same batch of different samples is little enough, and the ash condensation index can be applied as a measure of the ash condensation quality of cigarettes. Variation of ash condensation index is mainly caused by random errors in the production process. However, in order to ensure the measurement accuracy, multiple measurements could be taken to reduce the impact of random errors, and the maximum accuracy could be guaranteed of the ash condensation index in the application process.

Table 2 Standard deviation, mean value and variation coefficient of samples from the same brand

\begin{tabular}{lllllll}
\hline Samples & $1 \#$ & $2 \#$ & $3 \#$ & $4 \#$ & $5 \#$ & $6 \#$ \\
\hline $\begin{array}{l}\text { Standard } \\
\text { deviation }\end{array}$ & 1.30 & 1.01 & 0.67 & 0.42 & 1.85 & 1.54 \\
\hline Mean value & 93.64 & 98.06 & 98.46 & 97.66 & 90.38 & 88.74 \\
\hline $\begin{array}{l}\text { Variation } \\
\text { coefficient \% }\end{array}$ & 1.39 & 1.03 & 0.68 & 0.43 & 2.05 & 1.30 \\
\hline
\end{tabular}

It can be seen from Table 2 that the variation coefficient of different samples from the same brand is less than $3 \%$, which indicates that the ash condensation index of the same batch of different samples is little enough, and the ash condensation index can be applied as a measure of the ash condensation quality of cigarettes. Variation of ash condensation index is mainly caused by random errors in the production process. However, in order to ensure the measurement accuracy, multiple measurements could be taken to reduce the impact of random errors, and the maximum accuracy could be guaranteed of the ash condensation index in the application process.

\section{Advantages and Application Trend of Dust Analysis System}

In conclusion, the ash condensation index has the advantages of intuitionistic, accurate and analytical ability in measuring the ash condensation effect of cigarette paper. The variation coefficient data of the same batch samples is less than $3 \%$, and the ash condensation index is suitable in the evaluation of ash condensation of cigarette products.

At the same time, the ash condensation analysis system based on the principle of ash condensation index measurement has many advantages such as high image resolution, fast analysis speed and high precision, which is suitable as an important means of evaluating cigarette paper. The existing evaluation methods are basically subjective judgments and the appearance of the ash condensation score, which are not accurate and objective. How to use the evaluation method and the development of the ash condensation system as a measure of the effect of cigarettes as a more accurate, more effective tool is still a key technology to be solved in the future. At the same time, there is still room for improvement for the system in resolution and calculation accuracy, and the future system optimization should focus on how to optimize the relevant parameters and data processing and analysis ability more rationally.

\section{Conclusions}

(1)After comparing the ash condensation index and related ash appearance figures, the ash condensation index can be used as a measure of the effect of cigarette paper's ash condensation. The data obtained through ash condensation index are highly intuitive, accurate and analytical. The variationc oefficient of different sample data is less than $3 \%$.

(2) The ash condensation analysis system developed by authors has many advantages such as high image resolution, fast analysis speed and high precision, and it is suitable as an important means to evaluate and analyze the ash condensation of cigarettes, which could be combined with other evaluation methods as an effective tool for evaluating the ash condensation of cigarette paper.

\section{Acknowledgment}

Financial supports of China Tobacco Yunnan Industrial Co.,Ltd. (Grant Nos. 2017CP03\&2017CP01) are great acknowleged.

\section{References}

1. G.L. Yu. China Pulp \& Paper Industry, 6, 37 (2015)

2. J. Shen J, J.S. Li, X. R. Qian, et al. Carbo. Poly. 101, 769 (2014)

3. Y. Zhang, J.B. Zhan, Z.H. Yu, et al. China Pulp \& Paper, 35, 58 (2015)

4. G. Li, H.Y. Ding, J. Sun, et al. China Pulp \& Paper, 31, 32 (2012)

5. Y. Yu, J.B. Zhan, H. Wang, et al. 3rd ICEMEE IOP Conf. Series: Earth and Environmental Science, 1 26314 (2017).

6. G.Z. Li, P.J. Wang. Paper Chem. 4, 17 (2011)

7. J.X. Shen, J. Sun, W.Y. Xiao, et al. China Pulp \& Paper Indu. 33, 23 (2012)

8. Z.L. Zou, X. Hou. Heilongjiang Pulp \& Paper. 41, 35 (2013)

9. W.Song ,L. Li. Paper Chem. 4, 25 (2011)

10. P. Lei, S.Z. Shang, Y. Han, et al. China Pulp \& Paper, 33, 6 (2014) 
11. Y. Jing, J.Ma, M.Z. Zhou, et al. China Pulp \& Paper, 35, 69 (2016)

12. Z.G. Cheng, M.Q. Ye, S.X. Hu, et al. Toba. Sci. Tech, 2, 9 (2011)

13. Y. Zhang, J.B. Zhan, M.M. Miao, et al. China Pulp \& Paper, 36, 44 (2017). 